\title{
Evidence for boosting Mycobacterium tuberculosis- specific IFN- $\gamma$ responses at 6 weeks following tuberculin skin testing
}

\section{To the Editors:}

We read with great interest the paper by LEYTEN et al. [1] in the present issue of the European Respiratory Journal, which assessed whether the tuberculin skin test (TST) might affect the results of the QuantiFERON®-TB Gold (QFT-G), a bloodbased Mycobacterium tuberculosis-specific interferon- $\gamma$ release assay (IFNGRA).

This is an important topic in the UK with respect to the recently published National Institute for Clinical Excellence (NICE) guidelines [2] for the diagnosis and treatment of tuberculosis (TB). The guidelines state that if a suspected case of TB yields a negative initial TST then the subject should be retested at 6 weeks, at which point an IFNGRA may be used in conjunction with the TST. Experts within the field have previously been worried about the potential boosting effect of the TST on IFNGRA responses. However, to date, no study has been published in the UK.

As part of a project within our undergraduate medical degree course at Imperial College (London, UK), we conducted a similar project to that of LEYTEN et al. [1] using a smaller cohort of subjects. In addition, we looked at the effects of the TST administration not only on the QFT-G (Cellestis, Darmstadt, Germany), but also on responses in the other commercially available IFNGRAs, including T-SPOT.TB (T-SPOT; Oxford Immunotec, Abingdon, UK). Furthermore, we re-assessed responses at 6 weeks post-TST, since this time-point is in line with the NICE recommendations.

In total, we studied 10 subjects with no history of TB contact or disease. One subject was eventually discovered to have had previous exposure to TB. This subject's results were included in our study as a point of interest. Our results are summarised in table 1.

Overall our study concurs with that of LEYTEN et al. [1] for the early time-point. Out of the 10 subjects, nine had negative results at baseline and at $48 \mathrm{~h}$ in both assays used.

We found no evidence for boosting of QFT-G or T-SPOT responses from negative to positive following TST administration, if the IFNGRA blood sample was taken at the time of reading of the TST.

However, at the 6-week time-point we noticed discrepancies between initial and follow-up results for the QFT-G. Out of our 10 subjects, nine were QFT-G negative at the initial timepoint and of these, three (33\%) had become QFT-G positive at 6 weeks (table 1). We interpret these findings as evidence for boosting interferon- $\gamma$ responses through TST at 6 weeks.

\begin{tabular}{|c|c|c|c|}
\hline \multirow{2}{*}{$\begin{array}{l}\text { TABLE } 1 \\
\text { IFNGRA }\end{array}$} & \multicolumn{3}{|c|}{$\begin{array}{l}\text { Results of three time-points of the } \\
\text { QuantiFERON } N_{\mathbb{B}} \text {-TB Gold (QFT-G) and } \\
\text { T-SPOT.TB (T-SPOT) tests }\end{array}$} \\
\hline & Pre-TST & $48 \mathrm{~h}$ & 6 weeks \\
\hline \multicolumn{4}{|l|}{ QFT-G } \\
\hline Positive & 1 & 1 & 4 \\
\hline Negative & 9 & 9 & 6 \\
\hline \multicolumn{4}{|l|}{ T-SPOT } \\
\hline Positive & 1 & 1 & 1 \\
\hline Negative & 9 & 9 & 9 \\
\hline
\end{tabular}

These findings raise the question: why can a boosting effect be seen in the QFT-G over a 6-week-period and not in T-SPOT? We suggest that this may be because QFT-G uses the additional M. tuberculosis-specific antigen TB7.7, as well as the antigens early secreted antigenic target (ESAT)-6 and culture filtrate protein (CFP)-10, which are found in T-SPOT. The additional antigen may evoke a more persistent immunological response, which outlives the response of the common antigens and, hence, yields a positive QFT-G result at a later time-point.

Our results regarding the T-SPOT assay are in keeping with a study by RICHELDi et al. [3], who found that repeated TSTs over a period of 21 months did not induce a T-cell response to ESAT-6 or CFP-10 and, therefore, does not lead to falsepositive results in the T-SPOT assay thus maintaining high specificity.

Our results regarding a boosting effect on QFT-G after 6 weeks are in keeping with a study by CAUTHEM et al. [4], which showed that $30.9 \%$ of TST nonreactors showed a response at either 7 or 90 days after initial administration indicating a long-lived boosting effect. THOMSON et al. [5] also found that the boosting effect rarely occurs within 1 week, suggesting a reason as to why QFT-G was not positive at the 48-h timepoint.

We would like to encourage a larger study of the 6 week Mycobacterium tuberculosis-specific interferon- $\gamma$ release assay responses, to be conducted in subjects with and without prior tuberculosis exposure to confirm our findings. The potential boosting effect in the QuantiFERON ${ }_{\mathbb{R}}-\mathrm{TB}_{\mathrm{B}}$ Gold assay should be taken into account within the current National Institute of Clinical Excellence guidelines. 


\section{A. Naseer, S. Naqvi and B. Kampmann}

Academic Dept of Paediatrics, Imperial College, London, UK.

\section{STATEMENT OF INTEREST}

None declared.

\section{REFERENCES}

1 Leyten EMS, Prins C, Bossink AWJ, et al. Effect of tuberculin skin testing on a Mycobacterium tuberculosis-specific interferon- $\gamma$ assay. Eur Respir J 2007; 29: 1212-1216.

2 National Institute for Health and Clinical Excellence. Tuberculosis: clinical diagnosis and management of tuberculosis, and measures for its prevention and control. Clinical
Guideline 33. National Institute for Clinical Excellence, London, 2006.

3 Richeldi L, Ewer K, Losi M, Roversi P, Fabbri LM, Lalvani A. Repeated tuberculin testing does not induce false positive ELISPOT results. Thorax 2006; 61: 180.

4 Cauthen GM, Snider DE Jr, Onorato IM. Boosting of tuberculin sensitivity among Southeast Asian refugees. Am J Respir Crit Care Med 1994; 149: 1597-1600.

5 Thompson NJ, Glassroth JL, Snider DE Jr, Farer LS. The booster phenomenon in serial tuberculin testing. Am Rev Respir Dis 1979; 119: 587-597.

\section{Macrolides and bronchiolitis in infants}

\section{To the Editors:}

In a recent issue of the European Respiratory Journal, TAHAN et al. [1] presented their results on clarithromycin therapy in respiratory syncytial virus (RSV) bronchiolitis in 12 infants aged $\leqslant 7$ months, compared with nine controls. They concluded that clarithromycin had a beneficial effect assessed by: the length of hospital stay; the need for oxygen therapy; the need for $\beta_{2}$-agonist treatment; and the rate of readmission to hospital within the subsequent 6 months [1]. The authors explained their findings in terms of suppression of airway hyperresponsiveness, since there are experimental in vitro data indicating that macrolides may inhibit cholinergic stimulation of airway smooth muscle [2]. In addition, macrolides are known to have various anti-inflammatory effects [3]. Supporting the effects on airway inflammation and responsiveness, the 3-week clarithromycin treatment was associated with decreases in plasma interleukin (IL)-4, IL-8 and eotaxin concentrations [1].

Three questions are raised when reading the study by TAHAN et al. [1]. First, only one out of 12 children in the clarithromycin group, compared with four out of nine in the placebo group, was readmitted. However, this difference is not, at least when calculated with my computer, statistically significant. In addition, statistical calculations may not be justified when the numbers of cases are so small. Secondly, the need for $\beta_{2^{-}}$ agonists may not be a useful outcome measure, since these drugs are rarely effective in infantile bronchiolitis [4]. Thirdly, RSV antigen detection was the only microbiological method available. Normal white blood cell counts and C-reactive protein concentrations do not rule out infections caused by atypical intracellular bacteria, such as Bordetella pertussis, Chlamydia trachomatis and Simkania negevensis. There are two recent studies reporting that mixed RSV and $B$. pertussis infections are common in nonvaccinated infants [5, 6]. In some populations, $S$. negevensis involvement seems to be common in RSV bronchiolitis [7, 8].

Thus, the beneficial effects of clarithromycin in the acute phase of respiratory syncytial virus bronchiolitis may have been based on the coexistence of atypical bacteria. Clinically, the beneficial effects were marginal; on average, the duration of need for supplementary oxygen and the length of hospital stay decreased by 1.5 days [1]. As TAHAN et al. [1] stated, respiratory syncytial virus bronchiolitis is the most common lower respiratory tract infection in infancy. Due to the harmful effects of large-scale antibiotic use in infants, macrolide treatment, even if associated with some anti-inflammatory or bronchial reactivity decreasing effects, should be restricted only to the cases with proven or presumptive atypical bacterial aetiology of infection.

\section{Korppi}

Dept of Paediatrics, Tampere University and University Hospital, Tampere University, Tampere, Finland.

\section{STATEMENT OF INTEREST}

A statement of interest for M. Korppi can be found at www.erj.ersjournals.com/misc/statements.shtml

\section{REFERENCES}

1 Tahan F, Ozcan A, Koc N. Clarithromycin in the treatment of RSV bronchiolitis: a double-blind, randomised, placebocontrolled trial. Eur Respir J 2007; 29: 91-97.

2 Labro MT. Anti-inflammatory activity of macrolides: a new therapeutic potential? Antimicrobial Chemother 1998; 41: 37-46.

3 Culic O, Erakovic V, Parnham M. Anti-inflammatory effects of macrolide antibiotics. Eur J Pharmacol 2001; 249: 209-229.

4 American Academy of Pediatrics Subcommittee on Diagnosis and Management of Bronchiolitis. Diagnosis and management of bronchiolitis. Pediatrics 2006; 118: 1774-1793.

5 Crowcroft NS, Booy R, Harrison T, et al. Severe and unrecognised: pertussis in UK infants. Arch Dis Child 2003; 88: 802-806.

6 Korppi M, Hiltunen J. Pertussis is common in nonvaccinated infants hospitalized for RSV infection. Pediatr Inf Dis J 2007; 26: 316-318. 